\title{
Disseminated gonococcal infection: the importance of testing all mucosal sites
}

\author{
Keith Gunaratne MD, Wayne L. Gold MD, Peter E. Wu MD MSc
}

C Cite as: CMAJ 2020 June 15;192:E652. doi: 10.1503/cmaj.191361

A 27-year-old man with a history of HIV infection presented to hospital after 3 days of fever, night sweats and rash over his chest, abdomen and extremities (Figure 1). The patient had tenosynovitis in his left wrist but no swollen or tender joints. He reported that he had unprotected sex with a male partner, but he had no genitourinary symptoms. He had been adherent to antiretroviral medication and his viral load was undetectable. We suspected disseminated gonococcal infection. On further history, the patient clarified that he engaged in both receptive oral and anal intercourse.

We obtained a urine sample and the result was negative for gonorrhea on nucleic acid amplification testing (NAAT). Rectal and pharyngeal NAAT results were both positive for Neisseria gonorrhea. The patient was discharged the next day to complete a 1-week course of ceftriaxone ( $1 \mathrm{~g}$ administered intravenously daily). Results were negative for cultures of his blood.

At 1-month follow-up, the patient's symptoms had resolved and results from tests of cure with NAAT were negative. His serology result for syphilis was positive, for which we provided treatment with 1 intramuscular injection of benzathine penicillin $\mathrm{G}$.

Disseminated gonococcal infection complicated $0.4 \%-3 \%$ of gonococcal infections during the $1970-80 s^{1,2}$ Although rates declined thereafter, ${ }^{1,2}$ a recent resurgence has been attributed to oral-genital sexual practices among men who have sex with men (MSM). ${ }^{2}$ The classic presentation is a triad of dermatitis, arthralgias and tenosynovitis. ${ }^{2}$ Disseminated gonococcal infection can also present as a purulent mono- or oligoarthritis. ${ }^{1}$ The diagnosis can be missed because $50 \%$ of rectal and $90 \%$ of pharyngeal gonococcal infections are initially asymptomatic. ${ }^{1}$ The pharynx, in particular, seems to be a reservoir that contributes to transmission among MSM. ${ }^{2}$ Therefore, all potentially exposed mucosa should be screened for gonococcal infection. Nucleic acid amplification testing is the recommended diagnostic test owing to its high sensitivity (92\%-97.2\%) and specificity (96.1\%-99.8\%). ${ }^{3}$ Tests for co-infections such as syphilis and Chlamydophila should also be performed.

\section{References}

1. Vidaurrazaga MM, Perlman DC. A case of purulent gonococcal arthritis. IDCases 2019;19:e00662.

2. Belkacem A, Caumes E, Ouanich J, et al. Changing patterns of disseminated gonococcal infection in France: cross-sectional data 2009-2011. Sex Transm Infect 2013;89:613-5.

3. Public Health Ontario. Ontario gonorrhea testing and treatment guide, 2nd ed. Toronto: Queen's Printer for Ontario; 2018.

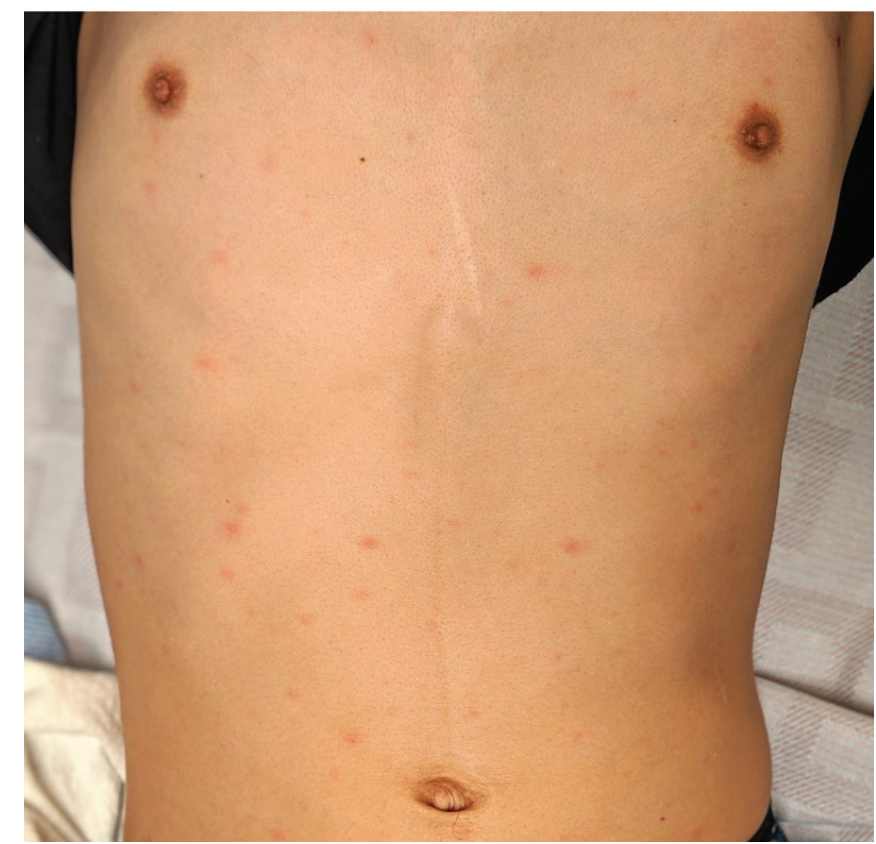

Figure 1: Multiple, discrete, erythematous and raised skin lesions on the chest and abdomen of a 27 -year-old man with disseminated gonococcal infection. A few scattered vesicular and pustular lesions were also found on his extremities.

Competing interests: None declared.

This article has been peer reviewed.

The authors have obtained patient consent.

Affiliations: Department of Medicine (Gunaratne, Gold, Wu), University of Toronto; Divisions of Infectious Diseases (Gold) and of General Internal Medicine (Gold, Wu), University Health Network, Toronto, Ont.

Correspondence to: Keith Gunaratne, keith.gunaratne@mail. utoronto.ca

Clinical images are chosen because they are particularly intriguing, classic or dramatic. Submissions of clear, appropriately labelled highresolution images must be accompanied by a figure caption. A brief explanation ( 300 words maximum) of the educational importance of the images with minimal references is required. The patient's written consent for publication must be obtained before submission. 